\section{Therapiefortschritte bei Epilepsie}

\author{
C. E. Elger ${ }^{1}$ \\ ${ }^{1}$ Klinik für Epileptologie, Universität Bonn
}

Die Epilepsie ist keine Erkrankung im eigentlichen Sinne, sondern hat eine Vielzahl verschiedener Ursachen, die vom genetischen bis hin zum entzündlichen Bereich reichen. Entsprechend problematisch ist daher die Diagnostik, die zum einen die Diagnostik der Anfallsphänomenologie als störendes Symptom für den Patienten beinhaltet und zum anderen einer Ursachensuche gilt, um die Notwendigkeit zusätzlich Behandlungen abzuklären, um den Verlauf der Erkrankung zu verbessern.

Eine der wichtigsten, nicht durch die Pharmaindustrie gesponserten Medikamentenstudien in der Epileptologie, ist die sogenannte SANAD-Studie, deren Ergebnisse gute Aussagen zur Effektivität der antikonvulsiven Medikation in verschiedenen Epilepsiesyndromen erlauben. Eine weitere wichtige Publikation liegt in Lancet Neurology im Jahre 2012 vor; sie beschäftigt sich mit den Risikofaktoren für das Therapieversagen bei über 2000 Patienten. Hier zeigt sich, dass die Zahl der Anfälle, eine neurologische Störung und das männliche Geschlecht negativ prädiktiv sind.

Als weiteres wichtiges Ergebnis konnte gezeigt werden, dass die Gabe von Valproinsäure in der Schwangerschaft zu einem niedrigen IQ bei den Kindern führt. Vergleichsdaten mit anderen Antikonvulsiva in der Schwangerschaft belegen dies in einer Analyse von 6-jährigen Kindern erneut deutlich. Ein wesentlicher Aspekt ist dabei, dass sich die Folsäure-Gabe in der Schwangerschaft positiv auf den IQ auswirkt. Dies unterstützt Studien, die sich mit Vitaminmangel-Zuständen bei Epilepsiepatienten beschäftigt haben. Es scheint so zu sein, dass eine dauerhafte Vitamintherapie bei Valproinsäure, Carbamazepin und Phenytoin angezeigt ist.

Eines der problematischsten Syndrome in der Epileptologie ist das Lennox-Gastaut-Syndrom. Es ist eine Enzephalopathie im Endstadium, die dominiert ist von Sturzanfällen, atypischen Absencen und anderen Anfallsformen. Der Cochrane Review von 2013 zeigte, dass es bisher keine Evidenz-basierte Therapie gibt, sondern dass bei komplizierter Therapie dieses Krankheitsbildes, vor allen Dingen Erfahrung und Vorwissen zu individuell akzeptablen Ergebnissen führen kann.
In der Beurteilung der Epilepsiechirurgie ist eine aktuelle Studie hilfreich; hier wurde versucht, eine frühe Epilepsiechirurgie mit einer Therapie durch Antikonvulsiva bei Temporallappen-Epilepsiepatienten zu vergleichen. Bereits bei einer kleinen Zahl war das Ergebnis der Temporallappenchirurgie so viel besser als die Weiterbehandlung mit Antikonvulsiva, dass es vor geplantem Studienende zum Abbruch der Studie kam.

Schließlich ist noch eine Studie erwähnenswert, bei der es um die prähospitale Therapie des Status epilepticus geht. Diese in den USA durchgeführte Studie verglich intravenöses Lorazepam mit intramuskulärem Midazolam. Das Ergebnis der Studie an 448 Patienten zeigte eindeutig eine Überlegenheit des intramuskulären Midazolams aufgrund der besseren Handhabe, sodass diese Therapie in der Notfallsituation des Status epilepticus als Gabe durch den erfahrenen Laien und nicht ärztliches Personal empfohlen werden kann.

Ein weiterer wichtiger Aspekt ist die Erfassung von epileptischen Anfällen beim Epilepsiepatienten. Zahlreiche Studien zeigen, wie unzuverlässig die Dokumentation in Patiententagebüchern ist. Da die Anfallsfrequenz für jegliche therapeutische Interventionen entscheidend ist, ist die Beantwortung der Zuverlässigkeit der Anfallsregistrierung für die Epileptologie von entscheidender Bedeutung.

Cook und Mitarbeiter haben in Lancet Neurology 2013 eine Arbeit publiziert, bei der sie mit Hilfe intrazerebral liegender Elektroden eine Anfallsvorhersage machen wollten. Dies gelang auch. Ein interessanter Zweitbefund der Publikation ist allerdings für die Epileptologie deutlich wichtiger. Die zuverlässige Registrierung über drei bis vier Monate mit intrazerebralen Elektroden zeigte deutlich, dass das Anfallstagebuch keinerlei Korrelation mit den objektiv gemessenen Anfällen hatte. Anfallstagebücher sind daher untauglich, einen Therapieerfolg zu messen.

Interessenkonflikt: Der Autor erklärt, dass er in keinem Interessenkonflikt steht.

\section{Bibliografie}

DOI http://dx.doi.org/10.1055/s-0033-1346713

Drug Res 2013; 63, Suppl. 1: S16-S16

(c) Georg Thieme Verlag KG Stuttgart · New York .

ISSN 2194-9379 